\title{
Line-strength indices and velocity dispersions for 148 early-type galaxies in different environments ${ }^{\star}$
}

\author{
J. Beuing ${ }^{1,2, \star \star}$, R. Bender ${ }^{2}$, C. Mendes de Oliveira ${ }^{1}$, D. Thomas ${ }^{2}$, and C. Maraston ${ }^{2}$ \\ ${ }^{1}$ Instituto Astronômico e Geofísico, Universidade de São Paulo, Avenida Miguel Stéfano 4200, 04301-904 São Paulo, \\ SP, Brazil \\ 2 Universitäts-Sternwarte München, Scheinerstr. 1, 81679 München, Germany
}

Received 30 July 2001 / Accepted 26 August 2002

\begin{abstract}
We have derived high quality line-strength indices and velocity dispersions for a sample of 148 early-type galaxies in different environments. The wavelength region covered by the observations $(\lambda \simeq 4600$ to $6600 \AA)$ includes the Lick/IDS indices $\mathrm{H} \beta, \mathrm{Mg}_{1}, \mathrm{Mg}_{2}, \mathrm{Mgb}, \mathrm{Fe} 5015$, Fe5270, Fe5335, Fe5406, Fe5709, Fe5782, NaD, $\mathrm{TiO}_{1}$ and $\mathrm{TiO}_{2}$. The data are intended to address possible differences of the stellar populations of early-type galaxies in low- and high-density environments. This paper describes the sample properties, explains the data reduction and presents the complete list of all the measurements. Most galaxies of the sample (85\%) had no previous measurements of any Lick/IDS indices and for 30\% of the galaxies we present first-time determinations of their velocity dispersions. Special care is taken to identify galaxies with emission lines. We found

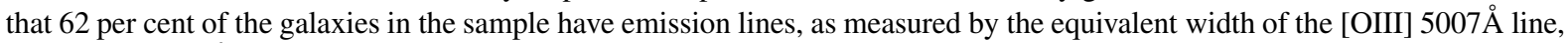
$E W[\mathrm{OIII}]>0.3 \AA$.
\end{abstract}

Key words. galaxies: elliptical and lenticular, cD - galaxies: abundances - galaxies: stellar content

\section{Introduction}

The stellar populations of early-type galaxies exhibit very homogeneous properties. This evidence comes from the Fundamental Plane (Djorgovski \& Davis 1987; Dressler et al. 1987; Bender et al. 1992; Jørgensen et al. 1993; Renzini \& Ciotti 1993; Saglia et al. 1993) and a number of scaling relations whose main characteristics are small internal scatters. Besides a well-defined correlation between color and magnitude (Bower et al. 1992), the Mg- $\sigma$ correlation (Bender et al. 1993) linking the stellar populations with the kinematical parameters of early-type galaxies also appears narrow. The studies mentioned above find that for bright cluster ellipticals the scatter in age is less than 15 per cent. This conclusion gets further support from the evolution of the above relations with redshift for cluster galaxies (Aragon-Salamanca et al. 1993; Dickinson 1995; Bender et al. 1996; Ziegler \& Bender 1997; Bender et al. 1998; van Dokkum et al. 1998; Jørgensen et al. 1999; Saglia et al. 2000).

\footnotetext{
Send offprint requests to: D. Thomas, e-mail: dthomas@mpe.mpg.de

* Tables 5 and 6 are only available in electronic form at the CDS via anonymous ftp to cdsarc.u-strasbg.fr $(130.79 .128 .5)$ or via http://cdsweb.u-strasbg.fr/cgi-bin/qcat?]/A+A/395/431.

They are also available via ftp at ftp.mpe.mpg.de in the directory people/dthomas/Beuing 02 or via WWW at ftp://ftp.mpe.mpg.de/ people/dthomas/Beuing02 .

$\star \star$ Visiting Astronomer, European Southern Observatory (ESO), La Silla, Chile.
}

The above findings are restricted to dense cluster environments. As theories of hierarchical galaxy formation predict that elliptical galaxies in the field will have less homogeneous properties and will be on average younger than their counterparts in clusters (e.g., Kauffmann et al. 1993; Cole et al. 1994; Thomas \& Kauffmann 1999, and references therein), it is particularly important to study early-type galaxies in different environments. Jørgensen et al. (1996) investigated the Fundamental Plane (FP) in 11 clusters of galaxies and found a small scatter of about 20 per cent. Moreover, they could not gather any evidence for differences in the FP depending on the environment. Also when leaving the clusters and going to less dense environments there is only weak and not yet compelling evidence that the homogeneity might disappear. Larson et al. (1980) find that the scatter of the color-magnitude relation in the field is slightly larger than that for cluster ellipticals. De Carvalho \& Djorgovski (1992) derive a larger scatter for the FP in the field compared to clusters. On the other hand, analyzing spectral features in the range $\lambda=4000-4400 \AA$, Rose et al. (1994) find that "the overall spectral type [...] of early-type galaxies does not vary with the environment". Interestingly, James \& Mobasher (1999), investigating the CO absorption feature at $2.3 \mathrm{~mm}$, find evidence for intermediate-age population only for galaxies in small groups, while the isolated early-type galaxies of their sample form a very uniform old population.

A further powerful tool to study stellar population properties of elliptical galaxies are measurements of absorption 
line-strength indices. In particular, a combination of age- and metallicity-sensitive indices allows for the derivation of average ages, metallicities, and $\alpha /$ Fe ratios (Worthey et al. 1992; Trager et al. 2000). Such line-strength indices are measured mostly for cluster galaxies in a number of studies in the literature (Peletier 1989; Worthey et al. 1992; González 1993; Davies et al. 1993; Carollo \& Danziger 1994; Fisher et al. 1995; Kuntschner 2000; Mehlert et al. 1998, 2000; Jørgensen 1999; Poggianti et al. 2001). In these studies, although the samples show a larger spread in ages than what is inferred from the scaling relations, (in particular massive) elliptical galaxies are found to have old mean ages. It should also be noted that composite stellar population models with a small metal-poor subcomponent can reproduce the Balmer absorption of all massive elliptical galaxies without invoking young ages (Maraston \& Thomas 2000). A further interesting result derived from the $\mathrm{Mg}$ - and $\mathrm{Fe}$-indices are the super-solar $\alpha / \mathrm{Fe}$ ratios, implying short star formation timescales (e.g., Matteucci 1994; Thomas et al. 1999).

Most of the ellipticals for which absorption line-strength indices are measured are in clusters. Our goal is to fill this gap in the literature, and present in this paper a sample of 148 nearby early-type galaxies which were observed spectroscopically in the wavelength range of $\lambda \simeq 4450-6650 \AA$. The following Lick/IDS absorption indices were measured: $\mathrm{H} \beta$, $\mathrm{Mg}_{1}, \mathrm{Mg}_{2}, \mathrm{Mgb}, \mathrm{Fe} 5015, \mathrm{Fe} 5406$, Fe5270, Fe5335, Fe5709, Fe5782, $\mathrm{NaD}, \mathrm{TiO}_{1}$ and $\mathrm{TiO}_{2}$. Their wavelength definitions can be found in Worthey et al. (1994). This paper presents the data which form the basis of our investigations.

The paper is organized as follows. Section 2 describes the sample properties. Section 3 explains the observations and the data reduction. The presence of emission is investigated in Sect. 4 , followed by a summary in Sect. 5 .

\section{Sample properties}

Our sample consists of 148 early-type galaxies which were observed spectroscopically. The selection of the sample galaxies is intended to complete a larger magnitude-limited sample of 530 galaxies with $B_{T} \leq 13.5 \mathrm{mag}$. The southern part of the sample observed here was compiled from a variety of catalogues including the list of Faber et al. (1989), the 3rd Reference Catalogue of Bright Galaxies (de Vaucouleurs et al. 1991, RC3) and the ESO Lauberts-Valentijn Catalogue (Lauberts \& Valentijn 1989, ESO-LV). The apparent blue magnitudes $B_{T}$ were taken from the RC3 and, when not available therein, from the ESO-LV. The absolute magnitudes $M_{B}$ were computed from the corrected $B_{T}^{0}$ magnitudes (see Table 5) taken first from the list of Faber et al. (1989) and subsequently from the RC3 and the ESO-LV using the heliocentric velocities derived here (see Table 5). A value of $H_{0}=50 \mathrm{~km} \mathrm{~s}^{-1} \mathrm{Mpc}^{-1}$ was used throughout the paper. The values of Faber et al. (1989) include additionally the $K$-correction following Pence (1976). The RC3 values are $K$-corrected following de Vaucouleurs et al. (1976, RC2). These latter corrections are usually small due to the low redshifts of the sample galaxies.

No detailed spectroscopy was previously available in the literature for most of the 148 galaxies studied here. For $85 \%$
Table 1. Galaxy types. $T$ : numerical type according to RC3 or ESO-LV, $T=-5$ stands for the interval $-5.5<T \leq-4.5$, for example. The fraction is given in per cent.

\begin{tabular}{lrrc}
\hline \hline Type & $T$ & number & fraction \\
\hline E & -5 & 47 & 31 \\
cD & -4 & 30 & 20 \\
E/S0 & -3 & 58 & 38 \\
S0 & -2 & 15 & 10 \\
later & $\geq-1$ & 2 & 1 \\
\hline
\end{tabular}

of the sample the Lick/IDS indices measured here are firsttime measurements and for $30 \%$ of the galaxies no velocity dispersion values were previously known. In particular, concomitant information on both the velocity dispersion and the line-strength indices was available for very few galaxies, specially for indices other than $\mathrm{Mg}_{2}$.

The histograms of $B_{T}$ and $M_{B}$ are shown in Fig. 1. The distribution of $M_{B}$ peaks at $M_{B} \approx-21.5$ mag with only little contribution from lower luminosity ellipticals with $M_{B}>-20$ and bright galaxies with $M_{B}<-23$ mag.

The observed sample comprises about 50 per cent elliptical and cD-type galaxies and 50 per cent E/S0 and S0 galaxies. A more detailed subdivision into morphological types can be found in Table 1.

The galaxies inhabit a variety of types of environments, from low- to high-density neighbourhoods. To describe the environmental density of a galaxy in our sample, we use the local surface density of galaxies $\left(N G_{T}\right)$, as given by the ESO-LV catalogue. It is derived as the number of the galaxies with an angular diameter larger than 1 arcmin on the ESO Quick Blue survey plates per square degree inside a radius of one degree around the considered galaxy. The considered galaxy itself is not included. Although being a projected surface density, it is a good indicator of the true volume density as discussed in ESO-LV. The density indicator $N G_{T}$ is available for 92 per cent of our sample. Another possible density indicator would be that supplied by the Nearby Galaxies Catalogue (Tully 1988) $\left(\rho_{\text {Tully }}\right)$. In this case the density is calculated using all companions brighter than -16 mag in the vicinity of the considered galaxy using redshift information. It reflects a volume density given in galaxies $\mathrm{Mpc}^{-3}$. Unfortunately $\rho_{\text {Tully }}$ is available only for a small fraction of our sample galaxies ( $\approx 25$ per cent), therefore we decided to use $N G_{T}$.

Figure 2 shows the distribution of the environmental densities, as given by the $N G_{T}$ parameter, for our sample galaxies. As a reference, approximate values of the environmental densities for Local Group (LG), Fornax and Virgo galaxies are indicated. Note that the quoted densities refer to the central regions of the respective environments. The transition from field/small group to cluster seems to be at $N G_{T} \simeq 9$ (ESO-LV). Taking this value as dividing line, 25 per cent of our sample galaxies are located in clusters and 75 per cent reside in environments with lower densities. 


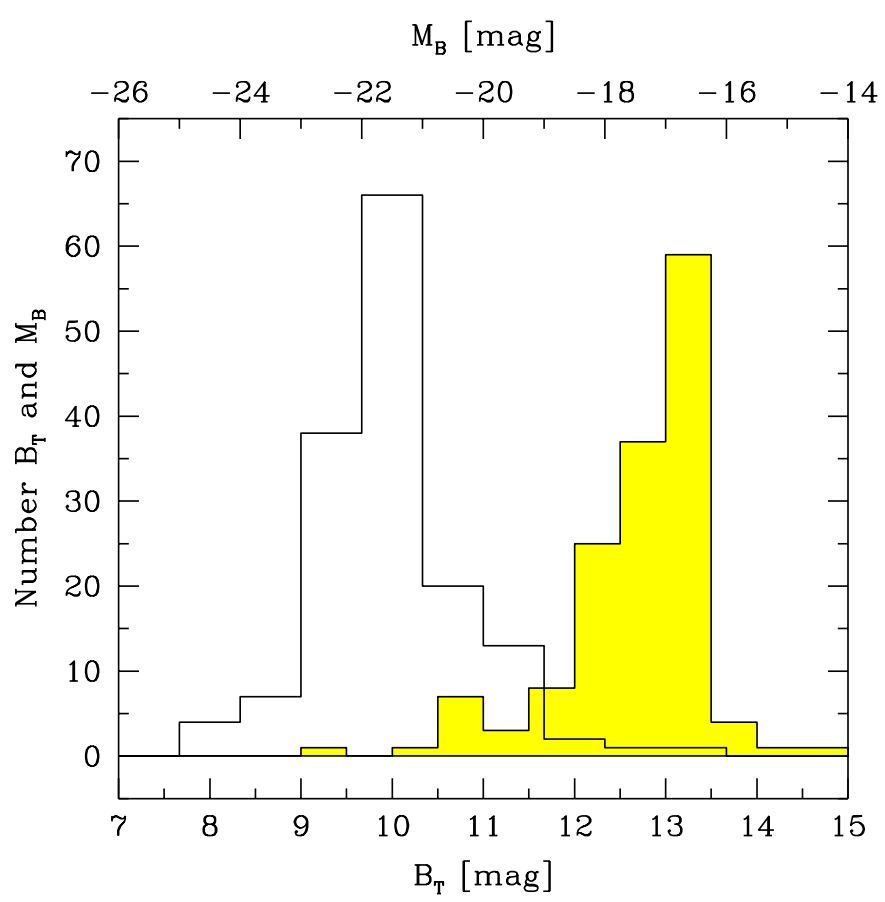

Fig. 1. Histograms of $B_{T}$ (shaded area) and $M_{B}$ (unshaded area) for the sample of 148 galaxies observed in this study. $H_{0}=50 \mathrm{~km} \mathrm{~s}^{-1} \mathrm{Mpc}^{-1}$ was used to calculate $M_{B}$.

\section{The data}

\subsection{Observations}

Longslit spectra were taken at the La Silla Observatory of the European Southern Observatory (ESO) during three observing runs from 12 to 19 of October 1995, 10 to 14 of April 1996 and 17 to 20 of October 1996. The Boller \& Chivens spectrograph mounted on the $1.52-\mathrm{m}$ telescope was used. A Ford aerospace 15-micron pixel CCD was used for the first run while a Loral/Lesser 15-micron pixel CCD was used in the other two subsequent runs. The slit width of 2 arcsec, in combination with all the other set-up parameters of the instrument, yielded a resolution of $\approx 100-120 \mathrm{~km} \mathrm{~s}^{-1}$, sufficient to measure the velocity dispersions $\sigma$ for most of the early-type galaxies observed. This choice of slit width ensured at the same time enough light throughput for good signal-to-noise ratio spectra with exposure times of $\approx 30 \mathrm{~min}$ and enough spatial resolution $(0.81$ arcsec $\mathrm{pix}^{-1}$ ) for a typical half-light radius of 30 arcsec. A sufficiently large part of the CCD was read out to allow accurate sky subtraction. No binning at read-out was applied to the data. The mean seeing was $\sim 1.3 \pm 0.2$ arcsec.

The observed wavelength covered the range from $\mathrm{H} \beta$ to $\mathrm{TiO}_{2}$ for a zero redshift galaxy in all the runs.

Bias frames were taken throughout the night. During the day time dark frames as well as high signal-to-noise dome flatfields were also made. The dome flatfields were produced before and after each night for the purpose of mapping pixel to pixel variations and possible sensitivity changes for example due to dust particles during the night. The median of 9-11 dome flats resulted in a noise level of the order of 0.1 per cent. In addition, sky flatfields were taken at dusk and dawn to

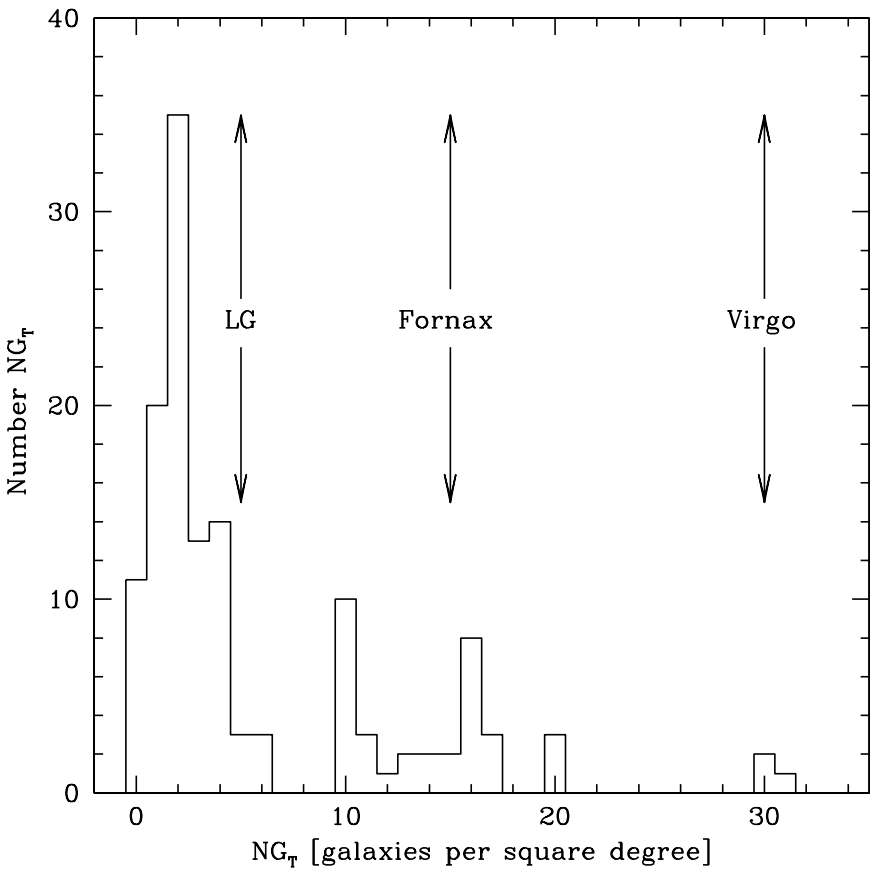

Fig. 2. Histograms of the environmental densities for our sample galaxies, defined by means of the $N G_{T}$ parameter (see the text). For reference, values of the environmental density of galaxies in or near the centers of the Local Group (LG) and the Fornax and Virgo clusters of galaxies are indicated.

correct the deviating slit illumination of the dome flatfields compared to sky images. This procedure ensured that the nightsky spectrum could be subtracted correctly.

A number of G- and K-giants stars were observed from the list of Worthey et al. (1994), in order to calibrate our linestrength index measurements to the Lick system, Further stars from the Bright Star Catalogue (Hoffleit et al. 1982) and from González (1993) were added, for the purpose of kinematical analysis (Table 2).

The stars were moved perpendicular to the slit back and forth in order to obtain a uniform illumination of the slit and high signal-to-noise ratios.

Helium-argon spectra were used to wavelength calibrate the spectra.

Total exposure times for galaxies ranged between $\approx 720-6600 \mathrm{~s}$. The spectra with longest exposure times usually resulted from two to three spectra with individual exposure times of about $\approx 1800-2400 \mathrm{~s}$.

During Runs 2 and 3 the slit was aligned with the major axis of the galaxy. The position angles of the galaxies (see Table 5) either came from the unpublished data of Bender et al. (1989) or were derived from Digitized Sky Survey plates. During Run 1 no alignment was performed to save valuable exposure time. The CCD in this run had a low quantum efficiency and it was decided to use the time which would have been needed to rotate the instrument to enhance the signal-to-noise ratio of the galaxy spectra. However, signal-to-noise ratios of the spectra taken during Run 1 are still considerably lower than those of Runs 2 and 3. 
Table 2. Observed stars. FAB: Faber et al. (1985), GON: González (1993) and BSC: bright star catalogue (Hoffleit 1982); Run ID's are reported in the last column.

\begin{tabular}{|c|c|c|c|c|}
\hline Name & $\begin{array}{l}\text { spectral } \\
\text { type }\end{array}$ & $\begin{array}{c}V \\
\text { mag }\end{array}$ & src & Run \\
\hline HD 036003 & K5 V & 7.64 & BSC & $1 \mathrm{a}, 1 \mathrm{~b}$ \\
\hline HD 064606 & G8 V & 7.44 & BSC & $1 \mathrm{a}$ \\
\hline HR 1015 & K3 III & 5.09 & FAB & $1 \mathrm{a}$ \\
\hline HR 2389 & K0 III & 5.22 & BSC & 3 \\
\hline HR 2429 & K1 III & 3.95 & FAB & 3 \\
\hline HR 2574 & K4 III & 4.07 & FAB & 3 \\
\hline HR 2701 & K0 III & 4.92 & FAB & 2,3 \\
\hline HR 2708 & G8 III & 5.46 & BSC & 3 \\
\hline HR 2778 & G9 III & 5.89 & BSC & 3 \\
\hline HR 2854 & K3 III & 4.32 & FAB & 2 \\
\hline HR 2970 & K0 III & 3.93 & FAB & 2 \\
\hline HR 3145 & K2 III & 4.39 & FAB & 2 \\
\hline HR 3242 & K1 III & 5.78 & BSC & 2 \\
\hline HR 3280 & K1 III & 5.07 & BSC & 2 \\
\hline HR 3392 & G9 III & 6.33 & BSC & 2 \\
\hline HR 3418 & K2 III & 4.44 & FAB & $1 \mathrm{a}$ \\
\hline HR 3427 & K0 III & 6.39 & FAB & $1 b$ \\
\hline HR 3428 & K0 III & 6.44 & FAB & $1 \mathrm{a}$ \\
\hline HR 3513 & G8 III & 5.87 & BSC & 2 \\
\hline HR 3728 & G6 III & 4.81 & BSC & 2 \\
\hline HR 3907 & G9 III & 6.35 & BSC & 2 \\
\hline HR 4287 & K0 III & 4.08 & FAB & 2 \\
\hline HR 6049? & K2 III & 6.14 & BSC & 2 \\
\hline HR 6136 & K4 IIIp & 5.39 & FAB & 1a \\
\hline HR 6159 & K7 III & 4.84 & FAB & $1 \mathrm{a}, 1 \mathrm{~b}$ \\
\hline HR 6501 & K0 III & 6.02 & BSC & 2 \\
\hline HR 6547 & K0 III & 5.93 & BSC & 2 \\
\hline HR 6590 & G7 III & 5.95 & BSC & 2 \\
\hline HR 6648 & G8 III & 5.84 & BSC & 2 \\
\hline HR 6651 & K1 III & 6.06 & BSC & 2 \\
\hline HR 6770 & G8 III & 4.64 & FAB & $1 \mathrm{a}, 1 \mathrm{~b}, 2,3$ \\
\hline HR 6998 & G4 V & 5.86 & GON & $1 \mathrm{a}, 1 \mathrm{~b}$ \\
\hline HR 7051 & A4 V & 5.06 & GON & 1a \\
\hline HR 7116 & K2 I & 4.83 & GON & $1 \mathrm{a}, 1 \mathrm{~b}$ \\
\hline HR 7149 & K2 III & 4.83 & BSC & 2,3 \\
\hline HR 7264 & F2 II & 2.89 & GON & $1 \mathrm{a}$ \\
\hline HR 7317 & K3 III & 6.06 & BSC & 3 \\
\hline HR 7429 & K3 IIIb & 4.45 & FAB & $1 \mathrm{a}, 1 \mathrm{~b}, 3$ \\
\hline HR 7430 & G9 IIIa & 5.12 & FAB & $1 b, 2,3$ \\
\hline HR 7456 & G0 Ib & 5.98 & GON & $1 \mathrm{a}$ \\
\hline HR 7965 & G7 III & 4.90 & BSC & 3 \\
\hline HR 7987 & K2 III & 5.35 & BSC & 3 \\
\hline HR 8229 & K0 III & 5.29 & BSC & 3 \\
\hline HR 8924 & K3 IIIV & 6.25 & FAB & $1 \mathrm{a}, 1 \mathrm{~b}$ \\
\hline
\end{tabular}

\subsection{Standard data reduction}

The bias frames showed temporal variations in the mean level as well as spatial gradients which were constant with time. Therefore a high signal-to-noise bias frame was constructed and subtracted from the science frames. The dark current for both chips was measured to be $\lesssim 1 \mathrm{e}^{-} \mathrm{pix}^{-1} \mathrm{hr}^{-1}$. Since most exposure times for the individual science frames were well below one hour, a subtraction of the dark current was omitted for all the frames.

In order to flat field the frames the median dome flat frames were corrected for nonuniform illumination using twilight flats. With this technique it was possible to flatfield the sky to better than 1 per cent in all cases.

Cosmic rays were removed with the MIDAS task FILTER/COSMIC. Care was taken not to remove real features. Afterwards the images were checked and remaining cosmic rays were removed manually.

The wavelength calibration of the data was done using the 10-15 strongest lines of the Helium-argon lamp. A fourth order polynomial was used to model the transformation from pixel to wavelength scale. Typical residuals are of the order of $\lesssim 0.05 \AA$, over the whole wavelength region covered. No significant deviation in the calibration was found when using the Helium-argon spectra taken before or after the galaxy exposure. The spectra were rebinned to a logarithmic wavelength scale to make the redshifting of lines independent of wavelength.

The subtraction of sky lines at the position of the galaxy spectrum was performed by linear interpolation using two windows of night sky not contaminated by spectra of neighbouring objects, one on each side of the galaxy spectrum. The windows were chosen to be as far away from the galaxy spectrum as possible in order not to subtract signal from the galaxy itself. This proved to remove most of the skylines in a sufficiently good manner. Remaining features for the strong skylines were removed manually.

The spectra were then rectified to lie exactly parallel to the dispersion direction. Afterwards, 1-dimensional spectra were extracted inside a range of $\pm 1 / 10$ effective radius along the observed axis which corresponds to a typical aperture of 2.8 arcsec times 2.0 arcsec. The values for the effective radii were taken from unpublished photometry. The effective radii derived for galaxies in common with the 7 Samurai sample are compared with the data of Faber et al. (1989) in Beuing (1998). A very good agreement is found. The values for the effective radii are listed in Table 5. For 24 galaxies (16 per cent of the sample) photometry is not available. In these cases we assumed a typical value of $\approx 30$ arcsec for the effective radius, which corresponds to the median effective radius of the sample (Beuing 1998). As index gradients in elliptical galaxies are shallow (Davies et al. 1993; Kuntschner 2000; Mehlert et al. 2000), the central values $\left(r<1 / 10 r_{\mathrm{eff}}\right)$ measured in this paper are not significantly affected by this choice within the errors.

\subsection{Velocity dispersions}

The Fourier-correlation-coefficient method (FCQ) of Bender (1990) was used to measure velocity dispersions. The FCQ method is based on the unfolding of the maximum of the galaxy-template star-correlation function with the maximum of the autocorrelation function of the template 
star. It minimizes the effect of template mismatching onto the measured value of the galaxy velocity dispersion.

The continuum subtraction was performed by first fitting the continuum of the template stars and the galaxies by a polynomial. With the spectra produced in this way a first approximate value of the velocity dispersion was determined. Afterwards, the spectra of the template stars were broadened with this approximate velocity dispersion to make them matching the galaxy spectrum as good as possible. In a next step, the continuum shape of the broadened template star was fitted by the same polynomial as was used for the galaxy spectrum and subtracted from the unbroadened template star spectrum. This template star spectrum, after the second iteration for continuum subtraction, was then used to determine the final velocity dispersion of the galaxy via the FCQ method. In this way, errors due to continuum subtraction are minimized.

Monte Carlo simulations were performed to choose the best suited template stars and to find the best polynomial fit for continuum subtraction following the subsequent recipe for all the template stars individually. A synthetic galaxy spectrum was constructed from a template star spectrum using the input parameters velocity dispersion and deviations of the line shapes from a Gaussian in form of Gauss-Hermite coefficients $\left(\mathrm{H}_{3}\right.$ and $\mathrm{H}_{4}$, see van der Marel \& Franx 1993) at a certain noise level. Afterwards, the template stars from which the synthetic galaxy spectra were constructed, were used to recover the input parameters through FCQ using the same scheme for continuum subtraction as described above. All different combinations of template stars and synthetic galaxy spectra were tested. The input parameters were thereby varied systematically in a plausible range for early-type galaxies. Each combination of the input parameters was performed with 100 different noise patterns of a certain signal-to-noise ratio. Only stars for which the input parameters could be recovered with a precision of better than a few percent (using the mean values of the 100 simulations) were selected to be suitable to derive the velocity dispersions of our sample galaxies. Moreover, by realizing 100 noise patterns also the error estimates of FCQ were calibrated, which are derived from photon statistics and read out noise. The template stars chosen in this way yielded very consistent results when applied to the real galaxy spectra (see below).

The velocity dispersions of Run 1 were derived calculating the mean of the values determined with the stars HR 3428 (K0 III), HR 6770 (G8 III), HR 3427 (K0 III) and HR 7430 (G9 III). They proved best suited from the Monte-Carlo simulations. The wavelength region used to determine the velocity dispersion lies between $4900 \AA$ and $5500 \AA$ (Fe5015 to $\mathrm{Fe} 5406) . \mathrm{H} \beta$ was not included to avoid additional template mismatching due to possible emission filling up $\mathrm{H} \beta$. For about $10 \%$ of the galaxies, additional observations were made for consistency check. In these cases, mean values weighted by the measurement errors were calculated. The mean FCQ error in the velocity dispersion in this run was $\sim 10 \mathrm{~km} \mathrm{~s}^{-1}$. The measurements using the different template stars agreed very well with each other. The standard deviation around the mean value was $\approx 8.5 \mathrm{~km} \mathrm{~s}^{-1}$. This value was of the same order as the mean FCQ error and thus indicates that the error estimate is correct and includes both the random errors as well as the systematic errors due the choice of the template star.

In Run 2 no significant difference of velocity dispersion measurements using all the different template stars was found. Hence mean values of all values were calculated. The mean FCQ error in velocity dispersion in this case was $6 \mathrm{~km} \mathrm{~s}^{-1}$. The same value was found for the standard deviation around the mean value calculated from all the different template stars, again indicating the validity of the error estimates. The lower error was mostly a result of the improved signal-to-noise ratio due to the new Loral/Lesser chip inserted into the spectrograph.

In Run 3 the Monte-Carlo simulations selected the stars HR 2429 (K1 III), HR 2701 (K0 III), HR 2708 (G8 III), HR 6770 (G8 III) and HR 7430 (G9 III). The mean FCQ measurement error was even lower than in Run 2, yielding a value of only $4 \mathrm{~km} \mathrm{~s}^{-1}$. The standard deviations around the mean values calculated from the different template stars mentioned above was $3 \mathrm{~km} \mathrm{~s}^{-1}$, again consistent with the FCQ error estimates.

The velocity dispersions together with the error estimates and heliocentric velocities are summarized in Table 5. The heliocentric velocities were calculated applying two corrections. The non-zero redshift correction for each star was determined using the Fe5335 line feature at $\lambda=5328.05 \AA$, because this line was almost free of blends and strong enough. The correction to the heliocentric system was calculated according to Stumpff (1980). The typical $1 \sigma$ error on the heliocentric radial velocity measurement is $\pm 20 \mathrm{~km} \mathrm{~s}^{-1}$.

Figure 3 shows the comparison of the velocity dispersions derived here with those from the catalogue of Prugniel \& Simien (1995). Excellent agreement is found. The Bisector (BS) zero-point shift proves to be not significant $(2.7 \pm$ $10 \mathrm{~km} \mathrm{~s}^{-1}$ ). Also the BS slope does not deviate from 1 significantly $\left(0.99 \pm 0.04 \mathrm{~km} \mathrm{~s}^{-1}\right)$. The scatter around the fitted line amounts to $23 \mathrm{~km} \mathrm{~s}^{-1}$, close to what expected from the quadratically combined errors in both directions $\left(22 \mathrm{~km} \mathrm{~s}^{-1}\right)$. This comparison sample however is biased to high $\sigma$ values.

Figure 4 shows another comparison with more recent results from the literature. The velocity dispersions given by Faber et al. (1997) are in excellent agreement with our measurements. A BS fit yields an insignificant zero-point shift of $5( \pm 16)$ and a slope of $1.01( \pm 0.08)$, consistent with 1 .

At $\sigma<200 \mathrm{~km}^{-1}$ we derive systematically higher values by $\sim 25 \mathrm{~km}^{-1}$ than Jørgensen (1997) and Kuntschner (2000). As Jørgensen (1997) and Kuntschner (2000) use fixed apertures, they sample a larger portion of the galaxy in low- $\sigma$ objects, which leads to lower mean $\sigma$ 's due to gradients.

All comparisons are summarized in Table 3.

\subsection{Line-strength indices}

Line-strength indices were measured in the Lick/IDS system as introduced by Faber et al. (1985) and extended and redefined by Worthey et al. (1994). We refer to the latter for the index definitions. The step by step procedure adopted to measure the line-strength indices was the following. 1) All the spectra were matched to the Lick resolution. 2) The line-strength indices 


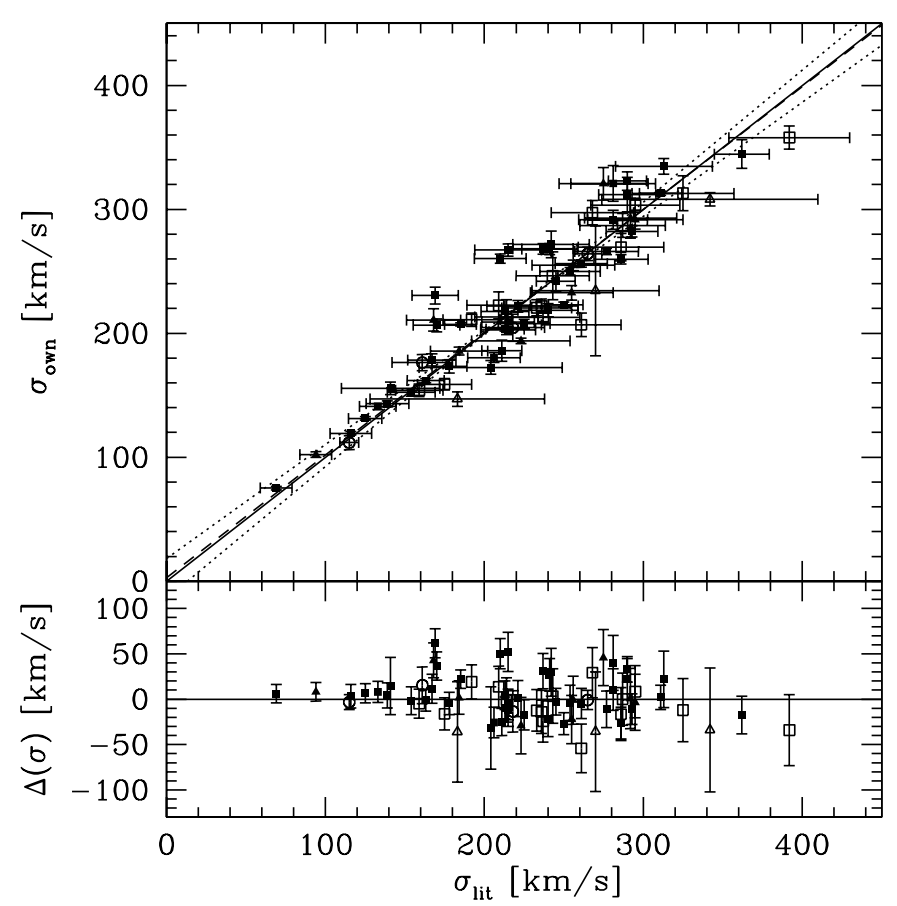

Fig. 3. Comparison of the velocity dispersions derived here $\left(\sigma_{\text {own }}\right)$ with the data given in the catalogue of Prugniel \& Simien (1995, PS). Filled squares represent values averaged over at least two different studies, filled triangles show the data of the 7 Samurai, open squares indicate data from DFB and open triangles refer to CPWSM, DON+94, TD81, LC, BER+94 (for references see PS). The long dashed line is a bisector least-square fit to the data, the dotted lines are the individual least-square fits in $X / Y$ and $Y / X$ direction. The bottom panel shows the residuals.

Table 3. Comparison of $\sigma_{0}$. Bisector fits resulting from comparison of our measurement with the data of Prugniel \& Simien (1995, PS 95), Faber et al. (1997, F97), Jørgensen (1997, J97) and Kuntschner (2000, $\mathrm{K} 00)$. ZP and SL stand for zero-point and slope respectively, $d(\mathrm{ZP})$ and $d(\mathrm{SL})$ for their errors.

\begin{tabular}{lcccc}
\hline \hline Source & $\begin{array}{c}\text { ZP } \\
\mathrm{km} \mathrm{s}^{-1}\end{array}$ & $\begin{array}{c}d(\mathrm{ZP}) \\
\mathrm{km} \mathrm{s}^{-1}\end{array}$ & SL & $d(\mathrm{SL})$ \\
\hline PS95 & 3 & 10 & 0.99 & 0.04 \\
F97 & -5 & 18 & 1.01 & 0.08 \\
J97 & 41 & 21 & 0.91 & 0.09 \\
K00 & 40 & 9 & 0.85 & 0.05 \\
\hline
\end{tabular}

were measured using the definitions of Worthey et al. (1994). 3) These raw measurements were corrected for the effects of velocity dispersion. 4) Zero-point to the Lick/IDS system were removed. Steps 1, 3 and 4 are explained in more detail below.

Step 1: our spectra were taken at the resolution of $4 \AA$ (corresponding to $\sigma \sim 100 \mathrm{~km} \mathrm{~s}^{-1}$ at $5100 \AA$ ) which is higher than that of the Lick spectra ( $8.6 \AA$ corresponding to $\sigma_{\text {Lick }} \sim 220 \mathrm{~km} \mathrm{~s}^{-1}$ at $\left.5100 \AA\right)$. Therefore, our galaxy spectra and template star spectra were broadened to the typical resolution of the Lick data by convolving them with a Gaussian of dispersion $\left(\sigma_{\text {Lick }}^{2}-\sigma_{\mathrm{i}}^{2}\right)^{1 / 2}$.

Step 3: the velocity dispersion of the stars in galaxies broaden the galaxy spectral features (in addition to the

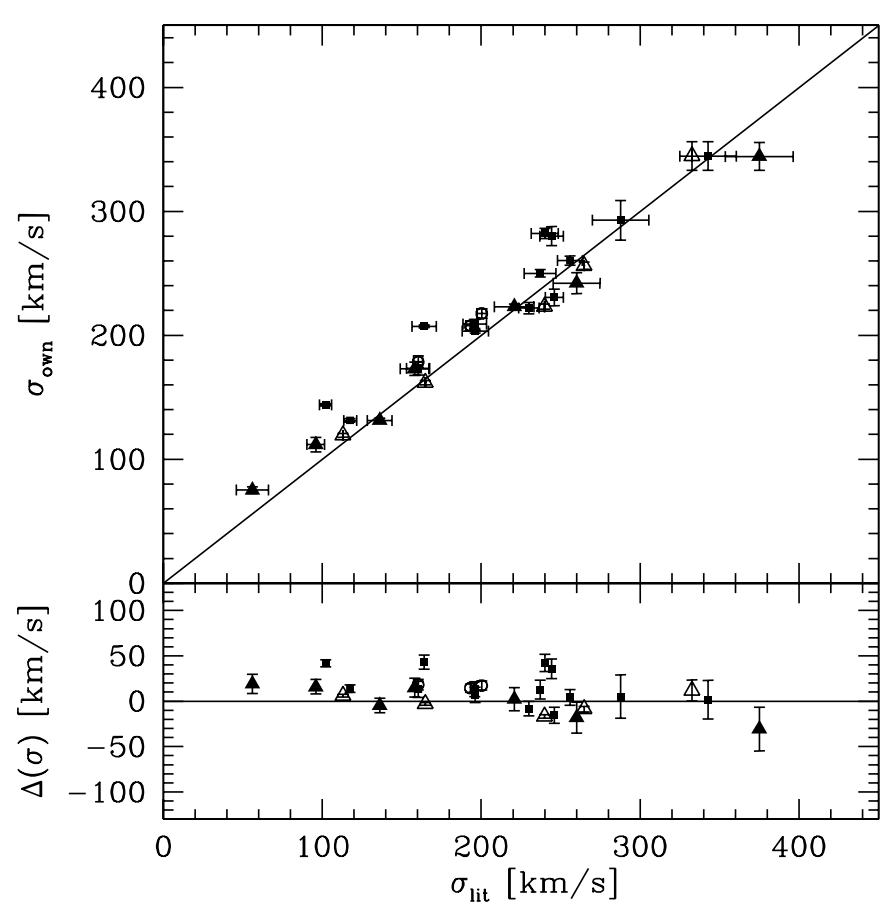

Fig. 4. Comparison of the velocity dispersions derived in this study $\left(\sigma_{\text {own }}\right)$ with data given in the literature. Filled squares represent values of Jørgensen (1997), filled triangles stand for the values of Kuntschner (2000), open circles mark values of González (1993), and open triangles represent values from Faber et al. (1997). The lower panel shows the deviations from the line of correspondence shown as a solid line in the upper panel.

instrumental broadening). As a result, line-strength indices decrease as their wings extend outside their index windows. In addition, other lines might reduce the continuum level by being broadened into the continuum windows. For this reason it is important to derive correction factors as a function of the velocity dispersion for each of the indices. These corrections are obtained adopting the standard procedure, as described e.g. in Davies et al. (1993).

In brief, template stars are broadened to simulate different velocity dispersions, ranging from 0 and $500 \mathrm{~km} \mathrm{~s}^{-1}$. Lick linestrength indices are then measured on these templates allowing to determine the velocity dispersion dependence of each index.

The measurement errors of the line-strength indices were derived as follows. For each continuum window and absorption line window, the read noise of the detector and the rms error from the electron count statistics were added in quadrature. Sky noise was never significant, as the central parts of the objects have higher surface brightness than the sky. Using standard error propagation the errors of the fluxes in the continuum windows and the line windows were combined to determine the error for each line-strength index. The reliability of the error estimate was checked by comparing repeated measurements of the same object and found satisfactory.

Step 4: zero-point corrections were finally applied to remove the offsets to the Lick system. These offsets were determined by measuring the indices of 3-8 stars per observing run (Table 2). 
Table 4. Offsets applied to calibrate on the Lick system.

\begin{tabular}{lrrr}
\hline \hline Index & Run 1 & Run 2 & Run 3 \\
\hline $\mathrm{Mg}_{1}$ & 0.082 & 0.014 & 0.016 \\
$\mathrm{Mg}_{2}$ & 0.060 & 0.005 & 0.006 \\
$\mathrm{Mg} b$ & 0.290 & 0.145 & 0.102 \\
$\mathrm{H} \beta$ & 0.123 & 0.012 & 0.008 \\
$\mathrm{Fe} 5015$ & 0.135 & -0.312 & -0.257 \\
$\mathrm{Fe} 5270$ & 0.034 & -0.153 & -0.079 \\
$\mathrm{Fe} 5335$ & 0.138 & 0.029 & 0.009 \\
$\mathrm{Fe} 5406$ & -0.074 & -0.092 & -0.119 \\
$\mathrm{Fe} 5709$ & -0.197 & -0.124 & -0.323 \\
$\mathrm{Fe} 5782$ & -0.195 & -0.120 & -0.192 \\
$\mathrm{NaD}_{\mathrm{TiO}}$ & -0.062 & -0.132 & -0.278 \\
$\mathrm{TiO}_{2}$ & -0.011 & -0.020 & -0.020 \\
\hline
\end{tabular}

The values for the line-strength indices derived in this work and in the literature (Worthey et al. 1994) are shown in Fig. 5. The offsets were obtained assuming slope 1 in each case. The offset is largest in case of Run 1. Except for Fe5709, the agreement between our measurements and the Lick values are good within the errors after zero-point corrections are applied. The adopted offsets are given in Table 4.

The galaxy database which has most objects in common with our sample is provided by Trager et al. (1998). Their NGC numbers are: 584, 596, 636, 1172, 1209, 1339, 1395, 1399, 1400, 1404, 1407, 2974, 3078, 3091, 3923, 5796, 5812, 6903, 7173, 7180. Figure 6 shows the comparison of the two data sets. In most cases the values compare well with each other within the error bars. The iron lines and the $\mathrm{TiO}_{1}$ band do not match very well. The reason for this stems mainly from the large errors of these indices in the Lick sample, which stretch the data distributions along the $x$-axis. The consistency of the iron line-strength indices derived by us with other sources in the literature supports this interpretation (see below).

The slightly larger scatter of our data taken in Run 1, which appears in $\mathrm{Mg}_{1}$ and Fe5015, is likely to be attributed to worse observational conditions compared to Runs 2 and 3 (see Sect. 3.1).

In Fig. 7 we compare our measurements with González (1993), Jørgensen (1997), Longhetti et al. (2000), and Kuntschner (2000).

On average these comparisons show better agreement than that with Trager et al. (1998). Here the various line-strength index systems seem to be on consistent scales inside the errors, although some nonzero systematic errors and zero-point offsets might be present.

Sources of systematic errors are: aperture and seeing effects, spectral sensitivity curves and possible applied calibration. The latter effect leads to different continuum levels and therefore affects the measurement process, especially for the indices for which the integration extends over a large range in wavelength. Particularly the scatter in the plots for $\mathrm{Mg}_{1}$ and $\mathrm{Mg}_{2}$ in Figs. 6 and 7 are larger than the error bars on the data most probably because of systematic differences in the continuum subtraction.
As a last remark on aperture effects, it should be stressed that index gradients are known to be rather small (see González 1993; Mehlert et al. 2000; Kuntschner 2000) and the errors we make by comparing measurements at different apertures is probably smaller than the error bars on the data. This is particularly true for the comparison with the data from Trager et al. (1998) plotted in Fig. 6.

The line-strength index measurements are given in Table 6 . To briefly summarize, the values were derived from nonfluxed-calibrated spectra, inside a range of $1 / 10$ of the effective radius of the galaxy along the major axis (except Run 1) corresponding to a typical aperture of 2.8 arcsec times 2.0 arcsec. They are corrected for the effects of velocity dispersion and calibrated to the Lick/IDS system by means of comparison stars using the data published by Worthey et al. (1994). The errors are derived from photon statistics and read out noise.

\section{Emission}

Emission lines which are present in the wavelength region covered by our spectra comprise $\mathrm{H} \beta(4861 \AA),[\mathrm{O} \mathrm{I}](6300 \AA$,

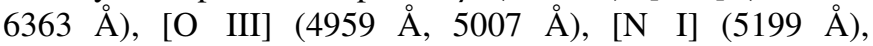
[N II] (5755 А, $6548 \AA, 6583 \AA), \mathrm{H}_{\alpha}(6563 \AA)$, Fe II (5270 $)$, [Fe VII] (5721 ̊), He I (5876 ̊) \& He II (4686 ̊), among others. Line-strength indices affected by these emission lines are $\mathrm{H} \beta, \mathrm{Fe} 5015, \mathrm{Fe} 5270, \mathrm{Fe} 5709, \mathrm{Mg}_{1}, \mathrm{Mg}_{2}, \mathrm{Mgb} \& \mathrm{NaD}$. In the case of $\mathrm{H} \beta$ and $\mathrm{Fe} 5270$ the emission lines are situated right on top of the line itself. The $[\mathrm{N} \mathrm{I}]$ emission line is found in the right continuum window of Mgb (see Goudfrooij \& Emsellem 1996 for a more detailed discussion). In the case of Fe5015 the two [O III] lines at $4959 \AA$ and $5007 \AA$ are found in both the left continuum and the line window. In all the other cases the emission lines are found very close to the edge of a window or just in the very narrow gap between the line window itself and the continuum window.

In all these cases the "true" value of the index may be influenced.

The strongest emission feature which can be identified unmistakably is the [O III] $5007 \AA$ emission line, which falls on top of the Fe5015 index. The [O III] $4959 \AA$ feature is found to be weaker in all spectra, consistent with theoretical expectations (Osterbrock 1989), often falling below the detection limit. Often connected to [O III] $5007 \AA$ is the emission in $\mathrm{H} \beta$, although line ratios can vary (Osterbrock 1989).

The strength of [O III] $5007 \AA$ was used to subdivide the sample into three classes. Galaxies of class " 0 " show no emission with $E W[\mathrm{O}$ III] $<0.3 \AA$, in class "1" the objects show weak/possible emission with $0.3<E W[\mathrm{O}$ III $]<0.5$ and in class "2" galaxies have the strongest emission lines, with $E W[\mathrm{O}$ III] $>0.5$. In general, for the intermediate class 1 , only [O III] $5007 \AA$ is detected, different from emission class 2, where several other emission lines are also detected. Typical examples are given Fig. 8. There is no evidence for emission lines in the spectrum of NGC 2986 which is therefore called a class "0" galaxy. The spectra of NGC 1549 and NGC 1316 contain instead emission lines. However, in NGC 1549 only a weak evidence for emission is found, located in the Fe5015 feature in the form of the [O III] $\lambda=5007 \AA$ line. This emission 

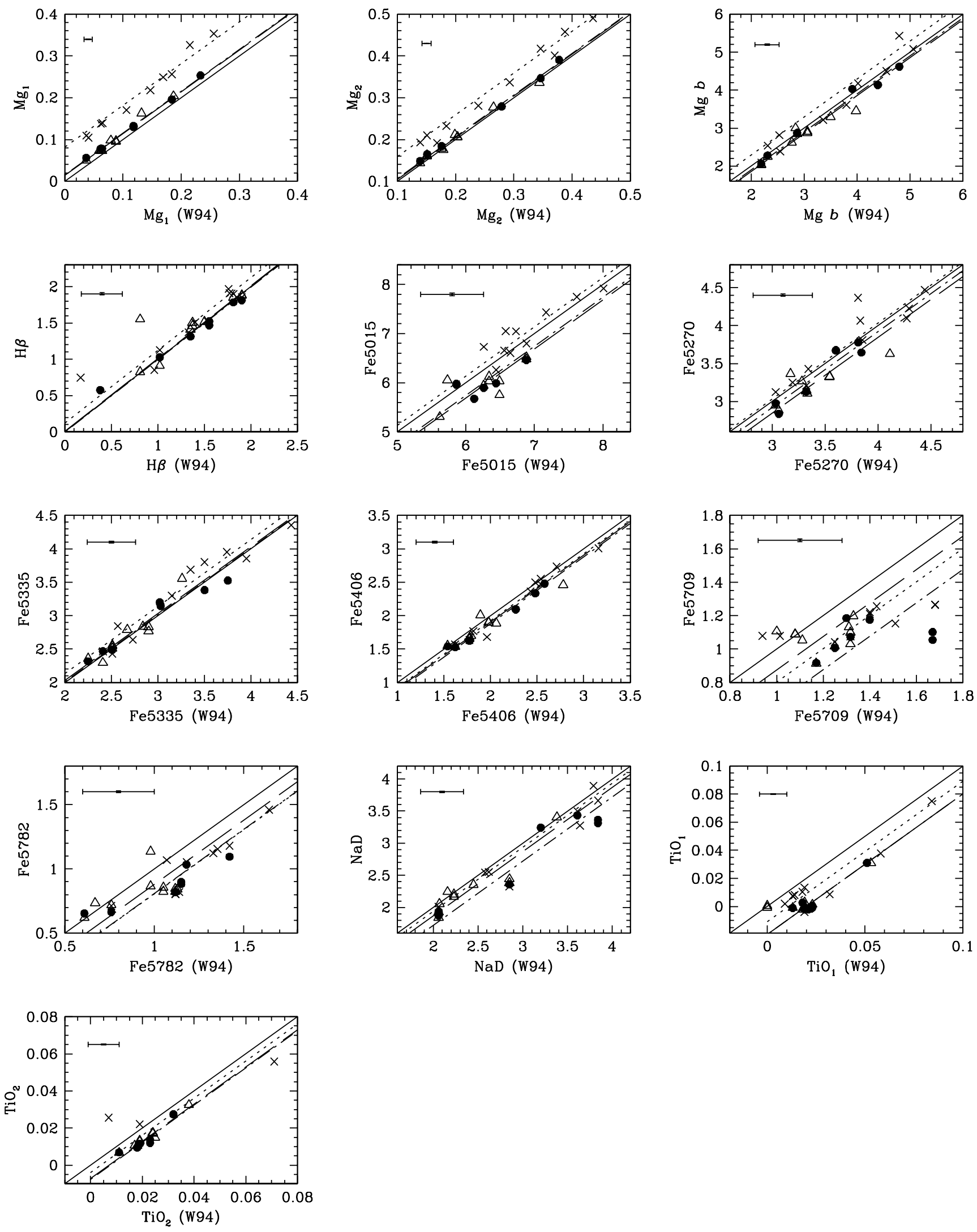

Fig. 5. Comparisons of the values for the line-strength indices derived in this work and in the literature (Worthey et al. 1994, W94). The offsets for the indices measured in each run were obtained assuming that the slope is 1 . The crosses and the dotted lines correspond to the data and best fit to the data of Run 1, respectively. Open triangles and a long-dashed line correspond to Run 2 while filled circles and the dot-dashed lines correspond to Run 3 . The line of correspondence is shown as a continuous line in each panel. 

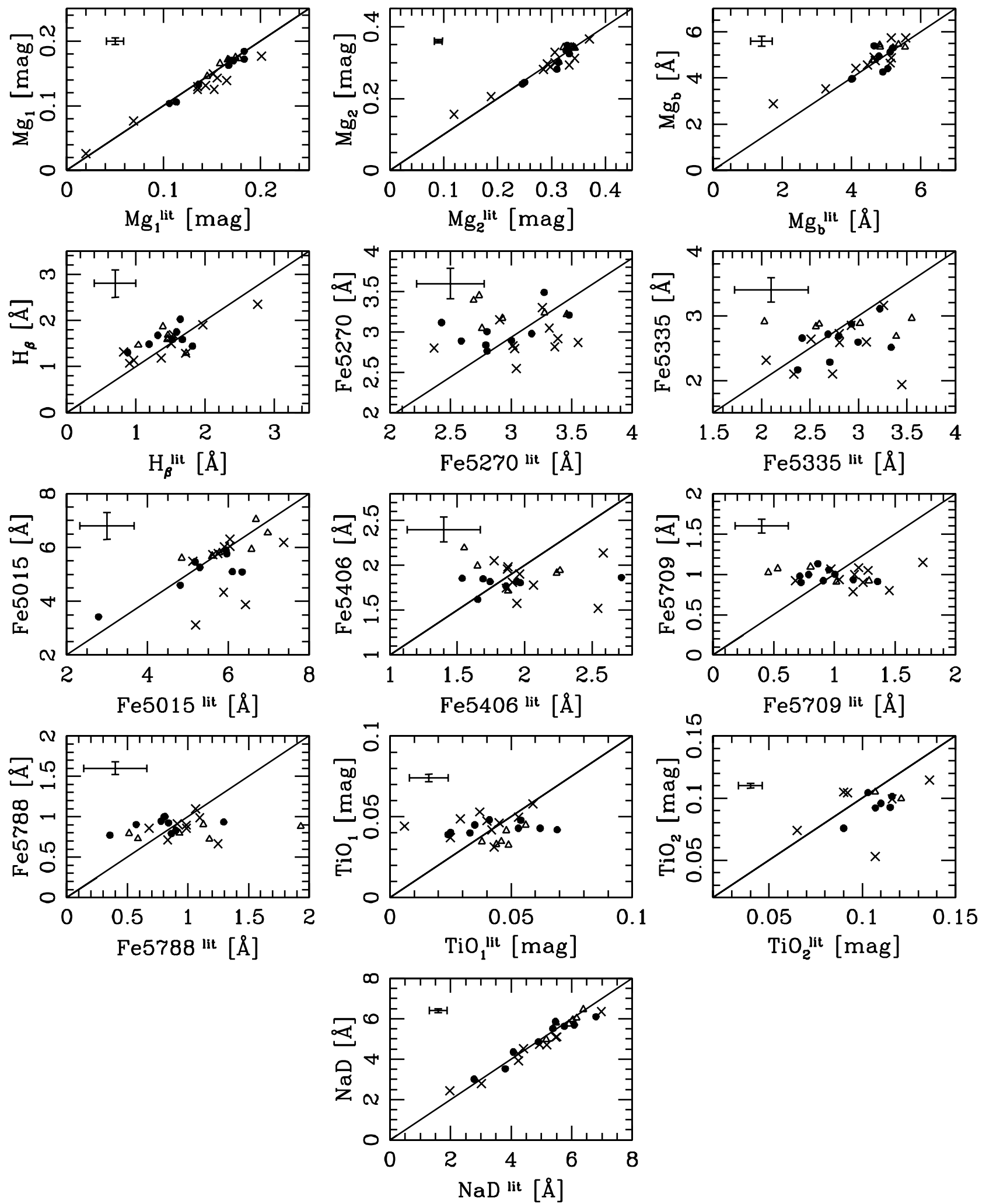

Fig. 6. Comparisons of the line-strength index measurements in common between our data and the data of Trager et al. (1998). Crosses, open triangles and filled circles refer to Run 1, Run 2 and Run 3, respectively. The error bars in the upper left corner of each panel indicate the median measurement errors. It should be stressed that our line-strength index measurements were calibrated exclusively by comparison stars. No further calibrations incorporating the galaxies in common with Trager et al. (1998) were made.

can not be verified at the positions of the other emission lines. for a galaxy with emission is NGC 1316. The emission is not Therefore the galaxy is classified as class " 1 ". A clear cut case only clearly visible in the [O III] $\lambda=5007 \AA$ line, but also at 

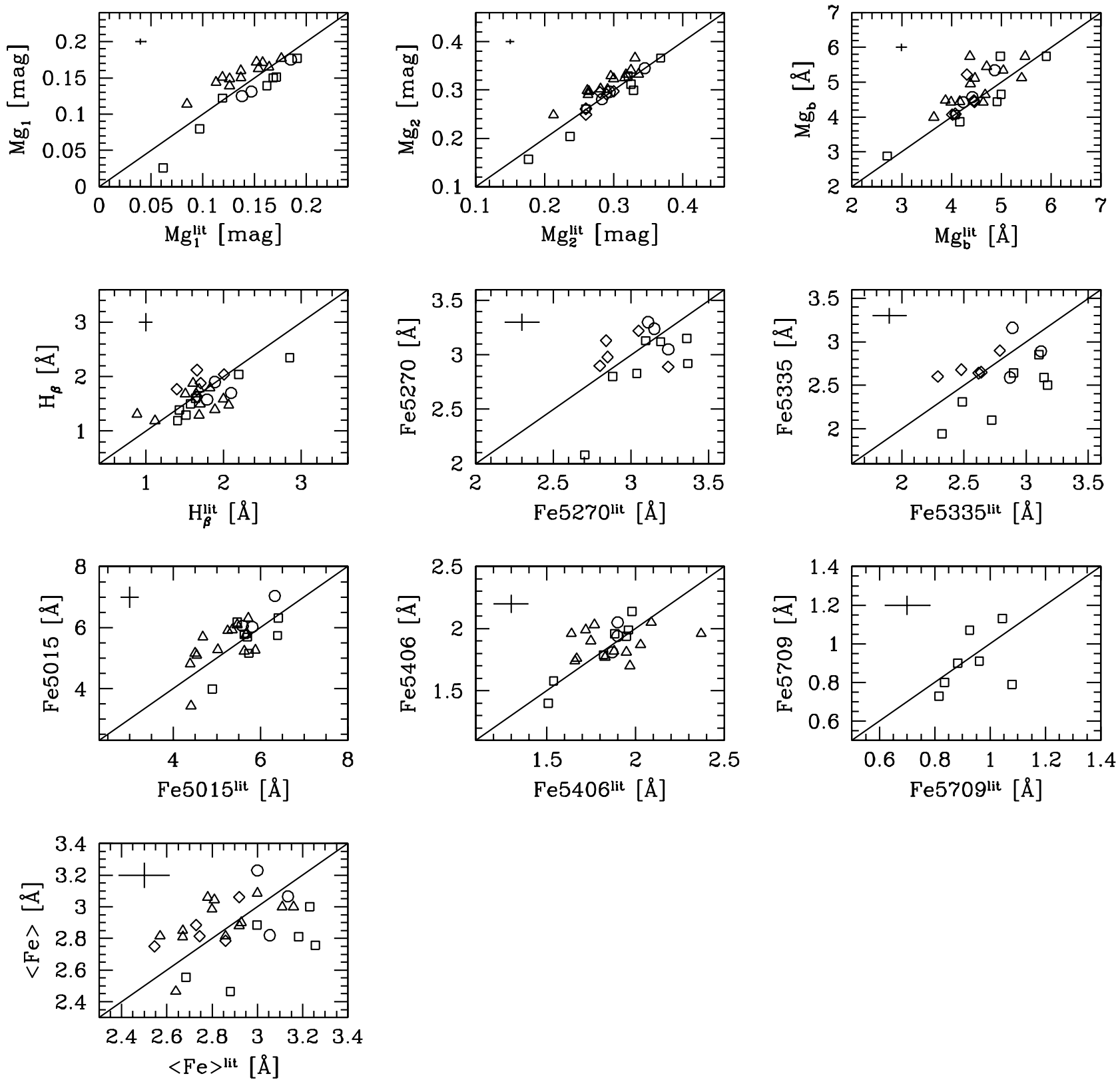

Fig. 7. Comparisons of the line-strength index measurements in common between our data and those from González (1993) (circles), Jørgensen (1997) (triangles), Longhetti et al. (2000) (diamonds), and Kuntschner (2000) (squares). The error bars in the upper left corner of each panel indicate the median measurement errors. The average $\mathrm{Fe}$ index $<\mathrm{Fe}>=(\mathrm{Fe} 5270+\mathrm{Fe} 5335) / 2$ is added (bottom-left panel), because Fe5270 and Fe5335 are not given separately in Jørgensen (1997).

$\lambda=4959 \AA$ ([O III]), $\lambda=4861 \AA$ (in the line center of $\mathrm{H} \beta$ ) and around $\lambda=6563 \AA\left(\mathrm{H}_{\alpha}\right)$. Therefore the galaxy belongs to class " 2 ".

Out of the 148 galaxies observed in this work, 56 objects (38 per cent) fall into class " 0 ", 45 objects (30 per cent) in class " 1 " and 47 objects (32 per cent) in class " 2 ". Altogether about 62 per cent of the galaxies show signs for some degree of emission. This result is consistent with the findings of Phillips et al. (1986), Caldwell (1984) and Goudfrooij et al. (1994), but it is lower compared to the result of Macchetto et al. (1996), who find that $\approx 79$ per cent of their sample galaxies have emission lines. This discrepancy may be explained by the fact that Macchetto et al. searched for line emission via narrow band imaging over the whole projected area of the galaxy while our data only refer to the galaxy centers.

We decided not to correct the line-strength indices for emission as the ratios between the various emission lines can vary significantly. Therefore, even a scaling of all emission lines, say, with the EW of [OIII], and subtracting a typical emission line pattern will not allow a consistent correction of all indices. However, the effect of emission is generally small. For the class 0 objects, the correction for emission is typically much smaller than the error bar. For class 1 objects, it is of the order of the error bar. Among the important indices, the $\mathrm{H} \beta$ index is most affected by emission. The correction can be estimated from González (1993) who gives $E W(\mathrm{H} \beta) \approx 0.7 E W([\mathrm{OIII}] 5007 \AA)$, 


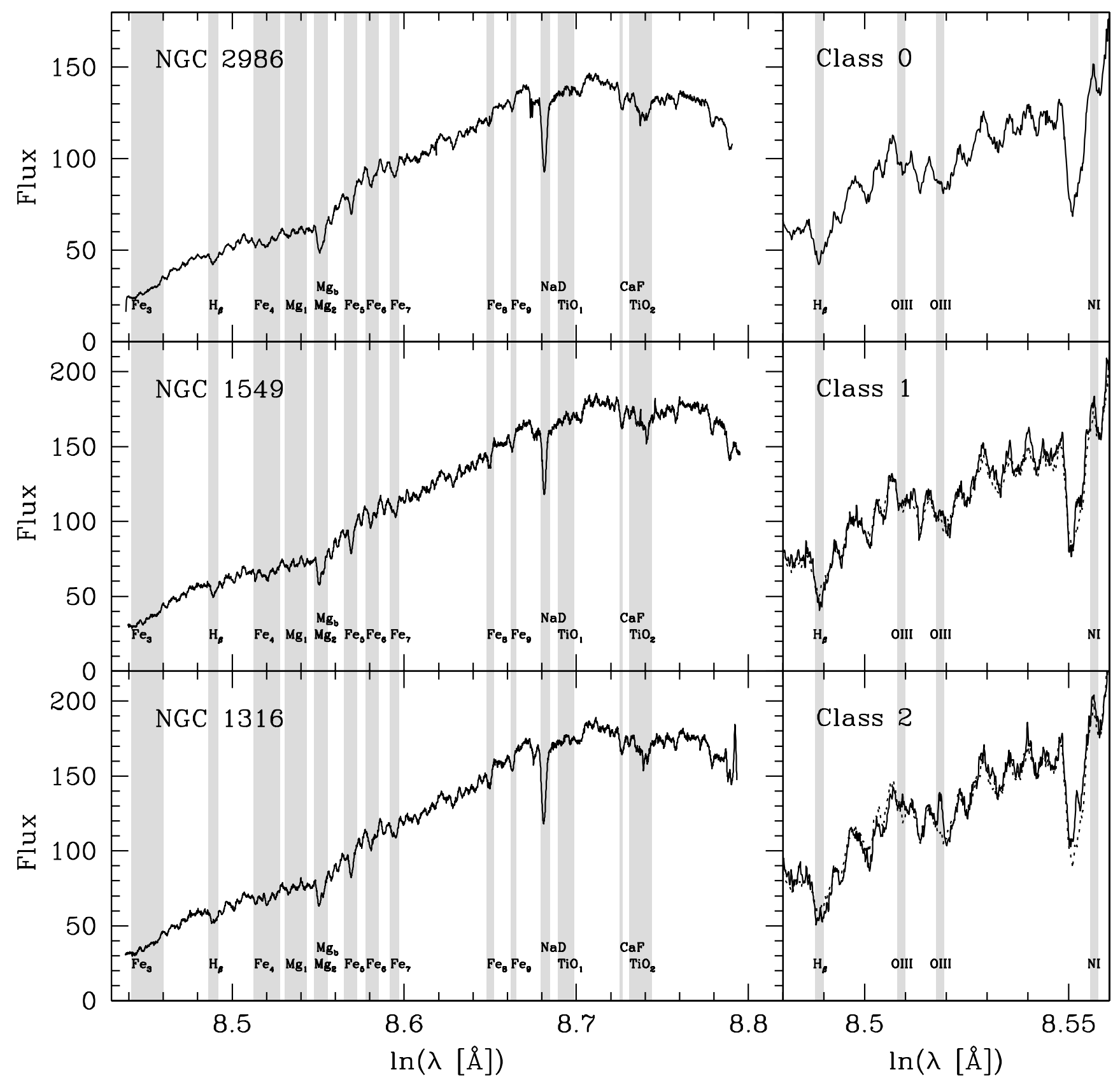

Fig. 8. Three examples of spectra with good signal-to-noise ratios plotted against the natural logarithm of the wavelength. Left panels: the full spectra transformed to zero redshift are shown. The flux is given in arbitrary units. Overlaid in light grey are the windows of the Lick/IDS indices measured in this work. Right panels: zoom of the spectra around the Fe5015 feature. In the upper left corner the emission class is indicated. Overlaid in light gray are the positions of emission lines. The dotted spectrum in the lower two panels is of NGC 2986 , rescaled to match the spectra of NGC 1549 and NGC 1316.

i.e. the correction is typically of the order $0.1 \AA$ for class 0 , $0.3 \AA$ for class 1 and $>0.4 \AA$ for class 2 objects.

\section{Summary}

This paper present the velocity dispersions and line-strength indices ( $\mathrm{H} \beta, \mathrm{Mg}_{1}, \mathrm{Mg}_{2}, \mathrm{Mgb}, \mathrm{Fe} 5015$, Fe5406, Fe5270, Fe5335, $\mathrm{Fe} 5709, \mathrm{Fe} 5782, \mathrm{NaD}, \mathrm{TiO}_{1}$ and $\mathrm{TiO}_{2}$ ) inside $1 / 10$ of the effective radius, for 148 early-type galaxies residing in different environments ranging from the field to clusters. The observational strategy and data reduction techniques are discussed. Velocity dispersions are derived using the Fourier-CorrelationCoefficient method of Bender (1990), whose power is to be only weakly sensitive to template mismatching. Line-strength index measurements are calibrated to the Lick/IDS system using stars in common with Worthey et al. (1994). Special care is taken to identify emission lines in the spectra of the sample galaxies. About 62 per cent of the total sample galaxies reveal some degree of emission, with $E W[\mathrm{OIII}]>0.3 \AA$, but only in 32 per cent of the cases emission is securely revealed $(E W[\mathrm{OIII}]>0.5 \AA)$ in several emission lines. 
The analysis of the absorption features in the spectra of the sample of galaxies presented here will be used for the investigation of the luminosity-weighted ages, metallicities and abundances for the bulk stellar populations of these galaxies.

Please note that Tables 5 and 6 are only available in electronic form at the CDS via anonymous ftp to cdsarc.u-strasbg.fr $(130.79 .128 .5)$ or via http://cdsweb.u-strasbg.fr/cgi-bin/qcat?]/A+A/395/431. They are also available via ftp at ftp.mpe.mpg.de in the directory people/dthomas/Beuing 02 or via WWW at ftp://ftp.mpe.mpg.de/people/dthomas/Beuing02.

Acknowledgements. This work was supported by the "Sonderforschungsbereich 375-95 für Astro-Teilchenphysik" of the Deutsche Forschungsgemeinschaft. CMdO acknowledges support also from FAPESP (Fundação de Amparo a Pesquisa do Estado de São Paulo) and the Alexander von Humboldt Foundation. DT and CM gratefully acknowledge the financial support from FAPESP and DAAD during their visit to the Universidade de São Paulo. We are grateful to the referee, Dr. Rampazzo, for the very careful reading of the fist version of the manuscript, and for the competent suggestions. The Digitized Sky Survey was produced at the Space Telescope Science Institute (ST ScI) under U. S. Government grant NAG W-2166. The images used were retrieved via Skyview, Goddard Space Flight centre, Greenbelt, MD 20771 301-286-7780. Skyview is supported by NASA ADP grant NAS 5-32068.

\section{References}

Aragon-Salamanca, A., Ellis, R. S., Couch, W. J., \& Carter, D. 1993 , MNRAS, 262, 764

Bender, R. 1990, A\&A, 229, 441

Bender, R., Capaccioli, M., Macchetto, F., \& Nieto, J.-L. 1989, ESO Messenger, 55, 6

Bender, R., Burstein, D., \& Faber, S. M. 1992, ApJ, 399, 462

Bender, R., Burstein, D., \& Faber, S. 1993, ApJ, 411, 153

Bender, R., Ziegler, B. L., \& Bruzual, G. 1996, ApJ, 463, L51

Bender, R., Saglia, R. P., Ziegler, B. L., et al. 1998, ApJ, 493, 529

Bernardi, M., Renzini, A., da Costa, L. N., et al. 1998, ApJ, 508, L43

Beuing, J. 1998, Ph.D. Thesis, Ludwig-Maximilians-Universität München

Bower, R., Lucey, J. R., \& Ellis, R. 1992, MNRAS, 254, 601

Caldwell, N. 1984, PASP, 96, 287

Carollo, C. M., \& Danziger, I. J. 1994, MNRAS, 270, 523

de Carvalho, R. R., \& Djorgovski, S. 1992, ApJ, 389, L49

Cole, S., Aragón-Salamanca, A., Frenk, C. S., Navarro, J. F., \& Zepf, S. E. 1994, MNRAS, 271, 781

Davies, R. L., Sadler, E. M., \& Peletier, R. F. 1993, MNRAS, 262, 650

de Vaucouleurs, G., de Vaucouleurs, A., \& Corwin, H. G. 1976, Second Reference Catalogue of Bright Galaxies (University of Texas Press, Austin) (RC2)

de Vaucouleurs, G., de Vaucouleurs, A., Corwin, H. G., et al. 1991, Third Reference Catalogue of Bright Galaxies (Springer Verlag, New York) (RC3)

Dickinson, M. 1995, in ASP Conf. Pro. 86, Fresh Views of Elliptical Galaxies, ed. A. Buzzoni, A. Renzini, \& A. Serrano (ASP, San Francisco), 283

Djorgovski, S., \& Davis, M. 1987, ApJ, 313, 59

Dressler, A., Lynden-Bell, D., Burstein, D., et al. 1987, ApJ, 313, 42

Faber, S. M., Friel, E. D., Burstein, D., \& Gaskell, C. M. 1985, ApJS, 57,711

Faber, S. M., Wegner, G., Burstein, D., et al. 1989, ApJS, 69, 763
Faber, S. M., Tremaine, S., Ajhar, E. A., et al. 1997, AJ, 114, 1771

Fisher, D., Franx, M., \& Illingworth, G. 1995, ApJ, 448, 119

González, J. J. 1993, Ph.D. Thesis, University of California, Santa Cruz

Goudfrooij, P., \& Emsellem, E. 1996, A\&A, 306, L45

Goudfrooij, P., Hansen, L., Jørgensen, H. E., \& Nœrgaard-Nielsen, H. U. 1994, A\&AS, 105, 341

Hoffleit 1982, Bright Star Catalogue, 4th edition

James, P. A., \& Mobasher, B. 1999, MNRAS, 306, 199

Jørgensen, I. 1997, MNRAS, 288, 161

Jørgensen, I. 1999, MNRAS, 306, 607

Jørgensen, I., Franx, M., \& Kjærgaard, P. 1993, ApJ, 411, 34

Jørgensen, I., Franx, M., \& Kjærgaard, P. 1996, MNRAS, 280, 167

Jørgensen, I., Franx, M., Hjorth, J., \& van Dokkum, P. G. 1999, MNRAS, 308, 833

Kauffmann, G., White, S. D. M., \& Guiderdoni, B. 1993, MNRAS, 264, 201

Kuntschner, H. 2000, MNRAS, 315, 184

Larson, R. B., Tinsley, B. M., \& Caldwell, C. N. 1980, ApJ, 237, 692

Lauberts, A., \& Valentijn, E. A. 1989, The Surface Photometry Catalogue of the ESO-Upsalla Galaxies (ESO, Garching bei München) (ESO-LV)

Longhetti, M., Bressan, A., Chiosi, C., \& Rampazzo, R. 2000, A\&A, 353,917

Macchetto, F., Pastoriza, M., Caon, N., et al. 1996, A\&AS, 120, 463

Maraston, C., \& Thomas, D. 2000, ApJ, 541, 126

Matteucci, F. 1994, A\&A, 288, 57

Mehlert, D., Saglia, R. P., Bender, R., \& Wegner, G. 1998, A\&A, 332, 33

Mehlert, D., Saglia, R. P., Bender, R., \& Wegner, G. 2000, A\&AS, 141,449

Osterbrock, D. E. 1989, Astrophysics of Gaseous Nebula and Active Galactic Nuclei (University Science Books, Mill Valey, CA)

Peletier, R. 1989, Ph.D. Thesis, Rijksuniversiteit Groningen

Pence, W. 1976, ApJ, 203, 39

Phillips, M. M., Jenkins, C. R., Dopita, M. A., Sadler, E. M., \& Binette, L. 1996, AJ, 91, 1091

Poggianti, B. M., Bridges, T. J., Mobasher, B., et al. 2001, ApJ, 562, 689

Prugniel, Ph., \& Simien, F. 1995, A compilation of internal stellar kinematics of galaxies

Renzini, A., \& Ciotti, L. 1993, ApJ, 416, L49

Rose, J. A., Bower, R. G., Caldwell, N., et al. 1994, AJ, 108, 2054

Saglia, R. P., Bender, R., \& Dressler, A. 1993, A\&A, 279, 75

Saglia, R. P., Maraston, C., Greggio, L., Bender, R., \& Ziegler, B. L. 2000, A\&A, 360, 911

Stumpff, P. 1980, A\&AS, 41, 1

Thomas, D., \& Kauffmann, G. 1999, in ASP Conf. Ser. 192, Spectrophotometric dating of stars and galaxies, ed. I. Hubeny, S. Heap, \& R. Cornett (San Francisco), 261

Thomas, D., Greggio, L., \& Bender, R. 1999, MNRAS, 302, 537

Trager, S. C., Worthey, G., Faber, S. M., Burstein, D., \& González, J. J. 1998, ApJS, 116, 1

Trager, S. C., Faber, S. M., Worthey, G., \& González, J. J. 2000, AJ, 119,164

Tully, R. B. 1988, Nearby Galaxies Catalogue (Cambridge University press, Cambridge)

van der Marel, R. P., \& Franx, M. 1993, ApJ, 407, 525

van Dokkum, P. G., Franx, M., Kelson, D. D., \& Illingworth, G. D. 1998, ApJ, 504, L17

Worthey, G., Faber, S. M., \& González, J. J. 1992, ApJ, 398, 69

Worthey, G., Faber, S. M., González, J. J., \& Burstein, D. 1994, ApJS, 94, 687

Ziegler, B. L., \& Bender, R. 1997, MNRAS, 297, 527 